Kampmann, Ronald; Sinzinger, Stefan:

Optical tweezers affected by monochromatic aberrations

Zuerst erschienen in:

DGaO-Proceedings. - Erlangen-Nürnberg : Dt. Gesellschaft für angewandte Optik. 117 (2016), Art. C6, 2 S.

ISSN: $\quad 1614-8436$

URN: $\quad$ urn:nbn:de:0287-2016-C006-7

URL: $\quad$ http://nbn-resolving.de/urn:nbn:de:0287-2016-C006-7

Download URL: http://www.dgao-proceedings.de/download/117/117_c6.pdf

[Download: 07.06.2017] 


\title{
Optical tweezers affected by monochromatic aberrations
}

\author{
Ronald Kampmann, Stefan Sinzinger \\ Fachgebiet Technische Optik, Institut für Mikro- und Nanotechnologien, Technische Universität IImenau \\ mailto:stefan.sinzinger@tu-ilmenau.de
}

\begin{abstract}
Standard optical tweezers are often built as inverted microscopes consisting of reflective, diffractive or refractive optical components. These optical elements are used to adapt the laser beam size, shape the beam profile and finally to generate the optical trap. Each optical component potentially suffers from various aberrations. In this contribution we investigate the effect of aberrations on the trapping performance.
\end{abstract}

\section{Introduction}

Nowadays optical tweezers (OT) are frequently applied in biological, physical and many other fields of research [1, 2]. For the majority of these optical systems classical optical components for beam adaption and deflection, as well as for focusing are used. Due to non-ideal optical properties the performance of optical tweezers differs often significantly from the theoretical predictions. The reasons are manufacturing related errors of the optical elements, geometric tolerances during the elements adjustment process and mounting up to approximations done in theory. In this work we focus on wavefront aberrations (WE) and nonsymmetric intensity profiles, both typical for nonideally adjusted optical systems. An optical force simulation based on geometrical optics approach [3] is used to theoretically explain the experimental results of optical trapping in the presence of optical aberrations.

\section{Theory}

As mentioned above, we use geometrical optics to describe optical forces [3]. Hereby a focused beam is approximated as a ray fan. If a ray interacts with a geometrical object like a sphere, it is divided into several components interacting multiple times with the object surface. These interactions lead to a momentum transfer onto the object. Summing up over all rays of the fan a net optical force can be calculated that acts on the object.

Using this procedure, WE [4] can be integrated into the force simulation by expressing them as optical path differences (OPD). Fig. 1 shows the OPD and WE caused by the aberration coma. As indicated, for on-axis points coma does not affect the optical system whereas field points located at the edge of the system are highly aberrated. These OPD values add a focal shift to an ideal system which means a broadening of the focal spot. Using this approach we consider optical aberrations in the optical force simulation. Furthermore, as input for the simulation the intensity distribution to be focused is required. Standard image files, which contain the beam profile, can be imported. That way non-ideal intensity distributions, as well as real measurement data can be addressed.

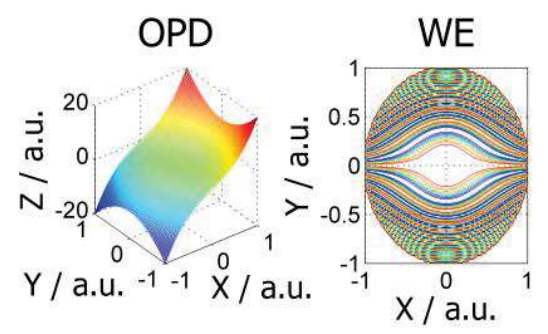

Fig. 1 Optical aberration coma.

\section{Experimental setup}

For our investigations we apply an optical system suited to trap particles in gaseous environment [5]. Optical trapping is realized by shaping and focusing a laser beam (green colored ray path in fig. 2). The observation (orange colored ray path) is implemented with a $45^{\circ}$ plane mirror and externally located imaging optics.

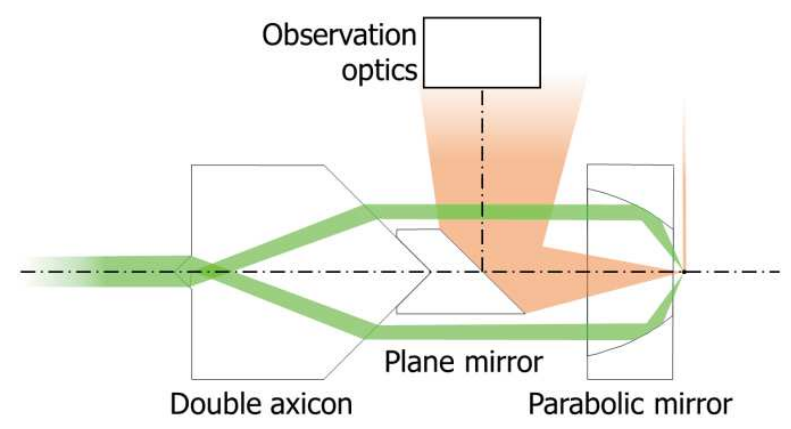

Fig. 2 Optical system for trapping (green) and observation (orange).

If all components are well adjusted, this setup provides nearly an ideal focal spot without aberrations. By specifically misaligning e.g. the double axicon (DA) optical aberrations can be introduced in a well-defined manner. For instance, a tilt of the DA results in a coma like aberration whereas a 
lateral shift causes a non-symmetrical ring distribution to be focused, see fig. 3 .

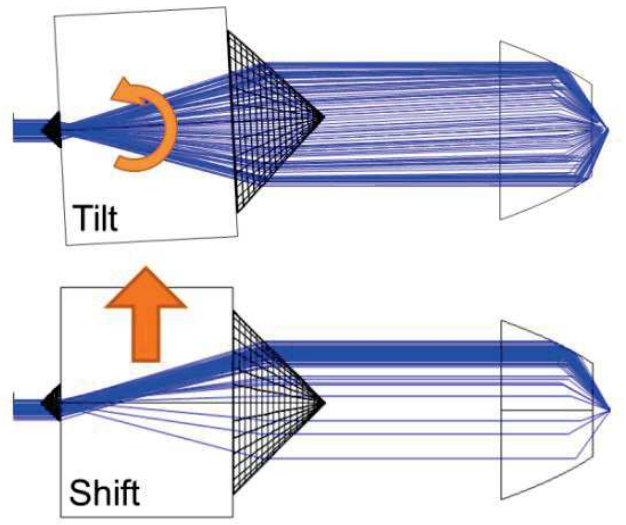

Fig. 3 Introduction of coma aberration (top) and nonsymmetric intensity profile (bottom).

First we use the well-adjusted optical tweezers to stably trap a spherical particle with $10 \mu \mathrm{m}$ in diameter made of fused silica. Next, while optically holding the particle, the system is de-adjusted and the behavior of the trapped particle is studied in the presence of aberrations. Finally, the spot of the aberrated optical tweezers is measured using a CMOS camera located at the focus plane. The recorded image, as well as the suspected kind of aberration is used as input for the optical force simulation and both, the simulation and the measurement result are compared.

\section{Results}

The double axicon was tilted to introduce coma like aberration while a particle was optically trapped. As a result, the particle moves upwards along a straight line of $100 \mu \mathrm{m}$ in length until it leaves the optical trap. By use of the optical force simulation this trajectory could be verified. Furthermore, the laser power necessary for experimentally trapping a particle using coma aberrated optical tweezers agrees with the simulation result.

The next aberration (no WE) was introduced by laterally shifting the double axicon to introduce an aberrated and inhomogeneous intensity distribution. Fig. 4 shows the optical trapping from side view whereas the bright area on the left represents the output aperture of the parabolic mirror. The trapping beam propagates in positive Z-direction and causes a heating of the gaseous surrounding of the particle. This finally result in a net force acting on the particle in upwards direction. Initially the trapped particle is located at the coordinates $Z=X=2 \mathrm{~mm}$. By shifting the double axicon in negative $X$-direction the particle starts a movement along a linear trajectory of more than $600 \mu \mathrm{m}$ in length, marked with the blue colored arrow in fig.4.

If all forces (drag, gravity) that act on the particle are considered, the trajectory described by the particle movement could be verified with the optical force simulation.

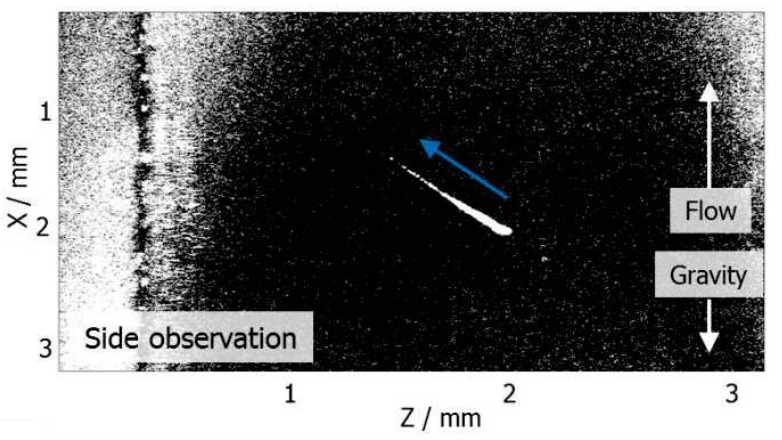

Fig. 4 Side view on trapped particle and particle movement (blue arrow) due to shift of DA.

As a consequence of this results and using additional experiments (investigation of spherical aberrations) the correct function of the optical force simulation could be confirmed. Hence, the effect of combinations of various aberrations on optical tweezers can now be studied from a theoretical point of view.

\section{Conclusion and Outlook}

We have shown how optical tweezers react on the introduction of two different types of optical aberrations. By comparing experimental results with the theoretical predictions calculated with an optical force simulation we found out that both results match nicely.

Current work is spent on improving the force simulation to increase the accuracy. Gaussian beams and diffraction effects are the next steps to be included. On the experimental side, the power spectrum method [6] will be applied to achieve more accurate position information compared to those obtained by the CMOS camera.

\section{References}

[1] I. Verdeny, A. Farre, J. Mas, C. Lopez-Quesada, E. MartinBadosa, and M. Montes-Usategui, "Optical trapping: a review of essential concepts," Opt. Pura Apl. 44, 527-551 (2011)

[2] K.C. Neuman, S.M. Block, "Optical trapping," Rev Sci Instrum.; 75(9): 2787-2809 (2004)

[3] A. Ashkin, "Forces of a single-beam gradient laser trap on a dielectric sphere in the ray optics regime," Biophys J., 61(2), (1992)

[4] W.T. Welford, "Aberrations of optical systems," (Adam Hilger, 1991)

[5] R. Kampmann, A. K. Chall, R. Kleindienst, and S. Sinzinger, "Optical system for trapping particles in air," Appl. Opt. 53, 777-784 (2014)

[6] K. Berg-Sørensen, and H. Flyvbjerg, "Power spectw..m nnal...in fan optical tweezers," Rev. Sci. In612 (2004) 